Chapman University

Chapman University Digital Commons

Physical Therapy Faculty Articles and Research

Physical Therapy

$4-2013$

\title{
Effects of Hemodialysis Therapy on Sit-to-Walk Characteristics in End Stage Renal Disease Patients
}

\author{
Rahul Soangra \\ Thurmon Lockhart \\ Virginia Tech Wake Forest University \\ John Lach \\ University of Virginia \\ Emaad M. Abdel-Rahman \\ University of Virginia
}

Chapman University, soangra@chapman.edu

Follow this and additional works at: https://digitalcommons.chapman.edu/pt_articles

Part of the Endocrine System Diseases Commons, Other Rehabilitation and Therapy Commons, and the Physical Therapy Commons

\section{Recommended Citation}

Soangra R, Lockhart TE, Lach J, Abdel-Rahman EM. Effects of hemodialysis therapy on sit-to-walk characteristics in end stage renal disease patients. Ann Biomed Eng. 2013;41:795-805. doi:10.1007/s10439-012-0701-6

This Article is brought to you for free and open access by the Physical Therapy at Chapman University Digital Commons. It has been accepted for inclusion in Physical Therapy Faculty Articles and Research by an authorized administrator of Chapman University Digital Commons. For more

information, please contact laughtin@chapman.edu. 


\section{Effects of Hemodialysis Therapy on Sit-to-Walk Characteristics in End Stage Renal Disease Patients}

\section{Comments}

This is a pre-copy-editing, author-produced PDF of an article accepted for publication in Annals of Biomedical Engineering, volume 41, in 2013 following peer review. The definitive publisher-authenticated version is available online at DOI: 10.1007/s10439-012-0701-6.

\section{Copyright}

Springer 


\title{
Effects of Hemodialysis Therapy on Sit-to-Walk Characteristics in End Stage Renal Disease Patients
}

\author{
Rahul Soangra ${ }^{1}$, Thurmon E. Lockhart ${ }^{1,2}$, John Lach ${ }^{3}$, and Emaad M. Abdel-Rahman ${ }^{4}$ \\ ${ }^{1}$ School of Biomedical Engineering and Sciences, Virginia Tech Wake Forest University, \\ Blacksburg, VA 24061, USA \\ ${ }^{2}$ Industrial \& Systems Engineering, Virginia Tech, Blacksburg, VA 24061, USA \\ ${ }^{3}$ Department of Electrical and Computer Engineering, University of Virginia, Thornton Hall, 351 \\ McCormick Road, Charlottesville, VA 22904, USA
}

${ }^{4}$ Division of Nephrology, University of Virginia, Charlottesville, VA 229084, USA

\begin{abstract}
Patients with end stage renal diseases (ESRD) undergoing hemodialysis (HD) have high morbidity and mortality due to multiple causes; one of which is dramatically higher fall rates than the general population. In spite of the multiple efforts aiming to decrease the high mortality and improve quality of life in ESRD patients, limited success has been achieved. If adequate interventions for fall prevention are to be achieved, the functional and mobility mechanisms consistent with falls in this population must be understood. Human movements such as sit-to-walk (STW) tasks are clinically significant, and analysis of these movements provides a meaningful evaluation of postural and locomotor performance in elderly patients with functional limitations indicative of fall risks. In order to assess the effects of HD therapy on fall risks, 22 sessions of both pre- and post-HD measurements were obtained in six ESRD patients utilizing customized inertial measurement units (IMU). IMU signals were denoised using ensemble empirical mode decomposition and Savistky-Golay filtering methods to detect relevant events for identification of STW phases. The results indicated that patients were slower to get out of the chair (as measured by trunk flexion angular accelerations, time to peak trunk flexion, and overall STW completion time) following the dialysis therapy session. STW is a frequent movement in activities of daily living, and HD therapy may influence the postural and locomotor control of these movements. The analysis of STW movement may assist in not only assessing a patient's physical status, but in identifying HD-related fall risk as well. This preliminary study presents a non-invasive method of kinematic measurement for early detection of increased fall risk in ESRD patients using portable inertial sensors for out-patient monitoring. This can be helpful in understanding the pathogenesis better, and improve awareness in health care providers in targeting interventions to identify individuals at risk for fall.
\end{abstract}

\section{Keywords}

Locomotion; Gait and posture; Fall risk; Gait initiation; Timed Get Up \& Go 


\section{INTRODUCTION}

Fall accidents pose a significant threat to older adults with End Stage Renal Disease undergoing HD therapy. In 2009, approximately 2.2 million nonfatal fall injuries were reported among older adults and were treated in emergency departments, and more than 581,000 of these patients were hospitalized. ${ }^{12}$ Falls contribute to approximately $95 \%$ of all hip fracture cases in the elderly population ${ }^{46}$; moreover, incidence of hip fractures in dialysis patients matched for age, gender and race is four times higher than that of general elderly population. ${ }^{45}$ As a result, incidence of falls in HD patients (1.18-1.60 falls/patientyear) were found to be much higher than community-dwelling elderly $(0.32-0.70$ falls/ person-year), $2,13,15,33$ affecting quality of life and requiring more supportive healthcare. ${ }^{33}$ ESRD patients on HD not only share the characteristics of the non-dialyzed elderly population that predispose them to falls, they also have additional risk factors specific to ESRD and/or HD, including: dialysis-related hypotension, myopathy, anemia, metabolic acidosis, dialysis disequilibrium syndrome, dialysis encephalopathy, catheter-related infection/sepsis, dialysis-related arrhythmias and post-dialysis fatigue ${ }^{8,20,44}$ Associated risk factors for fall in HD patients include a high prevalence of comorbidity, multiple drug therapy, ${ }^{15}$ cognitive impairment, ${ }^{32}$ low functional abilities compared with age-matched healthy persons ${ }^{24}$ and muscular strength degradation. ${ }^{34}$ Despite the myriad of research literature on fall risks specific to ESRD and/or HD, only a few studies to date have examined the dialysis process itself as a fall risk. ${ }^{34}$ In the context of HD-related fall risks, the limited reports provide veritable evidence that HD therapy can adversely affect strength (due to dialysis induced muscle changes) ${ }^{16,28,40,43}$ and movement coordination (influencing gait and posture $)^{6,18}$ thus increasing the likelihood of falls. In fact, similar studies confer that ESRD patients demonstrate a $66 \%$ reduction in comfortable walking speeds compared to their healthy, age matched counterparts. ${ }^{40}$ Therefore, considering ESRD patients generally undergo $4 \mathrm{~h}$ of HD therapy with an inter-dialysis interval of $22 \mathrm{~h}$ (three weekly HD sessions) and given the significant disparity in fall rates both before and after dialysis ((27\% predialysis vs. $73 \%$ post-dialysis), ${ }^{13,15}$ further studies are needed to better elucidate the mechanisms associated with HD and increased fall rate.

To quantify the mechanisms associated with HD therapy on fall risk between pre- and post$\mathrm{HD}$, baseline fall risk factors and differential effects of dialysis therapy (pre and post-HD) need to be determined. An objective method for assessing fall risk using body worn kinematic sensors and the Timed Up and Go (TUG) Test has been reported previously by several authors. ${ }^{21,47}$ Developed by Mathias et al., ${ }^{38}$ the TUG test is a well-known clinical test for mobility and fall risk, and is considered a reliable and valid test for quantifying functional mobility. ${ }^{31,39,48}$ For instance, increased TUG times are reported to be associated with high risk of falling in elderly patients with Parkinson's disease, stroke, and ESRD. ${ }^{1,34,42}$ In our previous investigations we have reported decline in plantar flexion muscle strength by $12 \mathrm{Nm}$ upon dialysis, and increased post-HDTUG $(p=0.02)$ time for ESRD patients. ${ }^{34}$ To our knowledge there has been no previous work committed to understanding the functional consequences of HD therapy and its effects on the activities of daily living. While TUG times provide an overall mobility index, they fail to distinguish pertinent information that is embedded in the components of the postural and locomotor tasks that contribute to slower overall movement time.

Sit-to-walk is a frequent movement in activities of daily living and its performance helps in both physical and mental assessment. STW is a complex sequential postural locomotor task that consists of two components: sit-to-stand (STS) and gait initiation (GI). Both tasks are merged at the point of "seat-off" in healthy young adults, where the overlap of STS and GI around "seat-off" is essential to exploit the inertial characteristics of both tasks. ${ }^{37}$ Nevertheless, the merging of these components at "seat-off" was not observed in some 
patients including the disabled population 11,17,30_i.e., completing the STS prior to initiating gait. Explanations of such distinct movement pattern include impaired balance, lack of strength leading to compensatory postural adaptation, lack of motor coordination, and fear of falling. ${ }^{3}$ As such, impaired timing of STW events and sequencing may place them at higher risk of falling. ${ }^{30}$ Furthermore, in order to assess the locomotion control behavior, ${ }^{9,22,23}$ the GI component of the STW task has been observed. The elderly population appears to have impaired STW motor performance ${ }^{10}$ and may be further exacerbated by HD therapy.

The purpose of this preliminary study was to compare intra-individual differences in STW parameters using a validated non-invasive mobile sensor technology to accurately and unobtrusively measure fall risks associated with HD therapy in the dialysis clinic. To accommodate the dialysis clinical setting, and bearing in mind the post-dialysis fatigue conditions of patients, inertial measurement units were utilized, in lieu of a motion capture system and forceplates, to detect postural and gait events. The overall aim of this investigation was to evaluate the effects of HD on STW parameters and fall risk assessment.

We postulate that the HD therapy process, a treatment for ESRD patients, is a risk factor for falls in this population and, will likely influence STW parameters, and subsequent event timing sequences. The present study will provide an initial assessment of the inherent effects of HD treatment on fall risks in this population for the first time, and may provide a better rationale for continuous monitoring in the future (i.e., $22 \mathrm{~h}$ post-HD period).

\section{METHODS}

\section{Participants}

Data was collected over the course of 5 months in the Kidney Center hemodialysis facility section of the Nephrology Division at the University of Virginia. Six ESRD patients (54 \pm 4 years) consisting of 4 females and two males, were tested with each HD treatment session lasting $4 \mathrm{~h}$. All participants had given written consent in accordance to the Virginia Tech and the University of Virginia IRB prior to participation. Blood pressure (BP) was monitored continuously throughout the dialysis treatments, while sitting and standing BP readings were taken immediately before and after each trial to check orthostatic BP. All patients were ambulatory, free of orthopedic injury, did not require any assistive devices, and were able to rise from a chair without assistance.

\section{Instrumentation}

TEMPO (Technology-Enabled Medical Precision Observation) which was manufactured in collaboration with the research team of the University of Virginia ${ }^{4}$ was used to assess posture-locomotor characteristics. TEMPO consists of MMA7261QT tri-axial accelerometers, a IDG-300 gyroscope ( $x$-and $y$-plane gyroscope) and ADXRS300 gyroscope ( $z$-plane uniaxial gyroscope). Data acquisition was carried-out using a Bluetooth-enabled laptop and a custom built LabView (National Instruments, Austin, TX) program ${ }^{4}$ with a sampling frequency of $128 \mathrm{~Hz}$, a frequency largely sufficient for human movement analysis in daily activities which occur in the lower bandwidth $(0.8-5 \mathrm{~Hz}){ }^{7}$

\section{Procedure}

Participants wore five IMU units (one on each lateral side of shank, and one on the sacrum, one on each wrist). The shank IMU units (TEMPO) were affixed at the lateral sides of each shank such that the $y$-axis corresponded to gravity and the $x$-axis corresponded to the anterio-posterior direction when participants stood in an anatomical neutral position. The IMU unit at the sacrum was positioned so that its $z$-axis aligned with the anterior-posterior 
direction and its $y$-axis aligned with gravity. The participants sat comfortably in a standard arm chair (fixed popliteal height $40 \mathrm{~cm}$ ), replete with arm and back support, with their thighs and feet parallel. To maintain consistency in all pre- and post-trials and to account for postdialysis fatigue, patients were instructed to sit all the way back (instructed to use backrest) and use the armrests for support to rise. Patients were asked to wait for an auditory signal before initiating movement. The patients were instructed to rise from the seat and walk at their self-selected pace to a target $3 \mathrm{~m}$ away from the chair. During the STW task, no restriction was placed on which foot to use for the first step in pre-HD trials, but all patients consistently used the same foot to initiate swing in post-HD trials.

\section{Data Processing}

The data was post processed using custom software written in MATLAB (MATLAB version 6.5.1, 2003, computer software, The MathWorks Inc., Natick, Massachusetts). empirical mode decomposition (EMD) ${ }^{25,26}$ is an adaptive time-frequency data analysis method; it can adaptively divide the IMU signals into different intrinsic mode function (IMF) components according to different time scales, and noise which primarily concentrates in the high-frequency component. IMU signals were decomposed adaptively into oscillatory components called IMFs using a process called sifting. The sifting process has two effects: (a) to eliminate riding waves; (b) to smooth uneven amplitudes. The traditional EMD involves the decomposition of a given signal $x(t)$ into a series of IMFs, through the sifting process, each with distinct time scales. ${ }^{26}$ Unlike wavelet decompositions the major advantage of the EMD is that the basis functions (mother wavelet; in case of wavelet transform) are derived from the signal $x(t)$ itself. Each IMF sequentially replaces then detail signals at a certain scale or frequency band ${ }^{19}$; ultimately the EMD picks out the highest frequency of oscillation that remains in the signal. An IMF adheres to two requirements: the number of extrema and the number of zero crossings are either equal or differ at most by one; at any point, the mean value of the envelope defined by the local maxima and the mean value of the envelope defined by the local minima is zero. The sifting is repeated several times to obtain a true IMF that fulfills the aforementioned requirements. The result of the sifting procedure is that $x(t)$ is decomposed into IMFs, $I M_{j}(t)=j=1,2$, $\ldots, N$ and residual $\iota_{\mathrm{N}}(t)$.

$$
x(t)=\sum_{j=1}^{N} I M_{j}(t)+r_{\mathrm{N}}(t)
$$

Let $C_{f}(t)$ be a deterministic IMF with the finite length $L$ and $I M_{j}$ the corrupted IMF with additive noise $b_{j}(t)$ :

$$
I M_{j}(t)=C_{j}(t)+b_{j}(t)
$$

Ensemble empirical mode decomposition (EEMD), is another approach which consists of sifting an ensemble of white noise-added signal and treats its mean as the true result. ${ }^{49} \mathrm{We}$ used EEMD-Golay denoising on IMU signals. The number of ensembles chosen was 100, with a 0.2 standard of deviation ratio of added noise to that of signal (Figs. 1a and 1b). First half of the IMF's containing high frequency noise are filtered using a Savitzky-Golay filter (polynomial order 3; number of frames as 41$)^{35}$ and subsequently reconstructed to obtain the denoised signals. 


\section{Variables and Analysis}

Comparisons between pre- and post-HD conditions were performed using paired $t$ test with an alpha set at 0.05 . In total eight postural transition and gait events were identified from the denoised STW component datum of the sacrum, right and left shank IMUs (Fig. 1a). These events include (E1) Initiation of STW, (E2) peak flexion angular velocity, (E3) seat-off event, (E4) peak extension angular velocity, (E5) swing toe-off, (E6) swing heel strike, (E7) stance toe-off and (E8) stance heel strike (Fig. 2). IMU's at wrist were used to reconfirm that all participants used arm-rest while rising from chair. Gait cycle was defined in accordance to previous research literature, ${ }^{10,11,29,30}$ with the initial stepping leg designated as swing and the second stepping leg designated as stance. The mean of three individual values from the three trials for each dependent variable during STW task were analyzed. The dependent measures of interest include time between detected events (E1-E8), phases and magnitudes of trunk flexion/extension angular velocity, initial flexion angular acceleration and trunk sagittal acceleration. Based on similar postural transition events and gait events, the STW phases were defined and validated by Kerr et al..$^{29,30}$ and Buckley et al. ${ }^{10,11}$ In absence of kinetic information from force plates and conflicts among previous studies ${ }^{11,29}$ for GI event detection, we have combined phase 2 (extension phase) and phase 3 (unloading phase) of the four phases defined by Kerr et al..$^{29,30}$ and Buckley et al. ${ }^{10,11}$ Previous studies on STW are laboratory based and required a camera system and force plate to evaluate center of mass (COM) vertical velocity and GI event. In order to develop more robust detection algorithms, we divided STW movement into three phases (Fig. 2) flexion momentum phase (phase 1), combined extension and unloading phase (phase 2), and stance phase (phase 3). Although distinct information for merging of STS and GI is completely lost but the overall time taken in trunk extension and unloading phase can be easily evaluated using IMUs with good robustness. The first phase of STW task is the flexion momentum phase, which encompasses the beginning of the movement (E1) until seat off (E3) (Fig. 2). In this phase high flexion velocity is generated and is later followed by seat unloading. Initiation of STW event (E1) is defined by denoised signals of IMU situated at the sacrum designated as the first local maxima before peak flexion angular velocity (global minima) (E2) in denoised Gyro X signals (Fig. 3a). The "seat-off" event (E3) is identified by the maximum acceleration in denoised Acc Z signals when STW signals are truncated to half of their total length (neglecting return datum of TUG test) (Fig. 3b). Additionally, denoised signals from the sacrum Gyro-X (across mediolateral axis) were used to acquire trunk peak flexion and peak extension angular velocities. Initial flexion angular acceleration of trunk is computed by fitting a line from the STW initiation event (E1) to peak flexion angular velocity event (E2) and finding its slope. The remaining two phases of STW are the combined extension and unloading phase (phase 2) and the stance phase (phase 3). The combined extension and unloading phase (phase 2) starts with development of extension velocity, followed by momentary stabilization, GI adjustments, and unloading; it comprises the seat-off event (E3) to the swing toe-off event (E5). Swing toe-off event (E5), is detected by the swing foot (starting foot) IMU situated at the shank. The first peak in denoised Gyro $\mathrm{Z}$ signals (across mediolateral axis) is maximum mid-swing angular velocity and the local minima to the left and right are designated as swing toe-off (E5) and swing heel-strike (E6) events respectively (Figs. 4a and 4b). Similarly, stance toe-off (E7) and stance heel-strike (E8) can be computed.

\section{RESULTS}

All six HD patients were able to complete the experiment trials without falling and without any postural hypotensive episodes. Although, all six patients experienced a drop in systolic blood pressure upon standing (between $10-15 \mathrm{mmHg}$ ), there was no report of postural hypotension episodes (i.e., none of them complained for any kind of dizziness or 
unsteadiness during upright standing). Percentage change in pre-dialysis systolic BP was $1.98 \pm 0.81 \%$ and for post-dialysis systolic BP was $0.58 \pm 0.9 \%$ for standing among the patients. Figure 5 shows mean $( \pm \mathrm{SD})$ changes of blood pressure during HD. A paired $t$ test between pre- and post-measures of STW movement determined that no significant differences existed in magnitude of peak anterior-posterior acceleration, peak angular flexion velocity, and peak extension angular velocity for pre- and post-HD conditions. After dialysis, significant differences were identified in the magnitude of initial flexion angular acceleration; average post-HD initial flexion angular acceleration was $2.48 \% \mathrm{~s}^{2}$ which are $23.2 \%$ less compared to that of pre-HD value $\left(3.23 \% \mathrm{~s}^{2}\right)$ (Table 1$)(p=0.02)$. While no significant differences were seen when comparing the magnitudes of peak flexion angular velocity, peak extension angular velocity and peak flexion acceleration, but the mean magnitudes of post-dialysis measures were smaller as compared to that of pre-dialysis measures.

Time to generate peak flexion velocity was defined as the time between the occurrence of peak flexion angular velocity event (E2) and the STW initiation event (E1) (Fig. 3a). Significant differences were detected in time to generate peak angular flexion velocity among pre- and post-HD patient STW trials $(p=0.03)$. Mean time for pre-HD trial was 0.47 $\mathrm{s}$, while post-dialysis was $0.79 \mathrm{~s}$. Similarly, significant differences in delay for generation of extension velocity were seen in the post-dialysis condition $(p=0.02)$. The mean time generation of peak extension velocity in pre- HD trial was $1.53 \mathrm{~s}$, while post-dialysis took $1.9 \mathrm{~s}$. Significant differences were also observed in time to STW completion for pre-HD and post-HD sessions $(p=0.04)$. Average post-HD STW completion time was $2.9 \mathrm{~s}$ which was approximately $15.2 \%$ more than pre-HD STW completion (2.46 s) (Table 2).

\section{DISCUSSION}

In this preliminary study we aim to understand the progression of movement from static posture to dynamic walking in pre-dialysis and post-dialysis conditions to assess fall risks associated with HD therapy. We found that HD therapy decreased STW parameters such as flexion acceleration prior to seat-off, and increased subsequent event timing sequences and thus may influence the risk of falls. Magnan ${ }^{37}$ suggested that balance during STW is dependent upon the flexion momentum generated prior to seat-off. The high flexion rate not only helps to rise out of the chair, it also assists in initiating a forward step. In this study, dialysis patients generated on average $23.2 \%$ less flexion angular acceleration prior to "seatoff" compared to values before dialysis therapy. These results suggest that there may be a greater risk for falls for individuals following HD therapy due to reduced momentum in the STW movement that may affect their balance.

Diminished STW and consequent balance complications can be symptomatic of decreased muscular strength, motor coordination, and inducement of post-dialysis fatigue.

Additionally, dialysis engenders change in biochemical parameters responsible for neuromuscular performance including (1) rapid changes in calcium concentration affecting muscle function; (2) acid-base balance affecting neuromuscular function. ${ }^{43}$ The change in calcium ion concentration may produce considerable variability in muscle function, leading to a concomitant decrease in force production and resistance to fatigue. ${ }^{5}$ Acidosis, on the other hand, may affect actomyosin interaction and potentially slow down the steps in crossbridge cycle by altering the $\mathrm{Ca}^{++}$sensitivity of muscle. ${ }^{14}$ The decreased $\mathrm{Ca}^{++}$sensitivity elicits less force production at the same level of activation. ${ }^{41}$ These muscle changes may be responsible for diminished STW performance in post-dialysis condition.

STS, which is also a component of STW, depends on a multitude of sensorimotor, balance, psychological factors ${ }^{36}$ and combined lower limb strength measures, i.e., hip extension, 
knee extension and ankle plantar flexion strength. ${ }^{34}$ The overall degradation in performance of STW was evident in the significant difference in time taken to generate peak flexion angular velocity with mean time extending from 0.47 to $0.79 \mathrm{~s}$ after HD. This delay in required flexion velocity generation (about $68 \%$ more transition time) could possibly degrade postural balance after seat off. Further, significant differences were observed in time taken for the trunk to reach peak flexion velocity (event E2), seat-off (E3) and peak extension velocity (E4). The slow velocity generation following dialysis therapy, can be attributed to an inability to maintain timing sequence in postural transitions.

A reduction in a person's ability to simultaneously lift and transfer the body from quiet sitting to walking, can be a critical limiting factor for the quality of life. More information on STW movement characteristics may help in understanding these post-dialysis functional problems. Healthy young adults typically take about $0.43-0.53 \mathrm{~s}$ in phase I (movement initiation to seat-off) and healthy older adults take nearly $0.41-0.59 \mathrm{~s},{ }^{10}$ yet we determined that HD participants took $0.9-1.18 \mathrm{~s}$ prior to dialysis and $1.18-1.54 \mathrm{~s}$ post-dialysis in the same phase. Likewise, phase I durations were significantly different for pre- and post-HD trials. This delay in phase I duration suggests that older HD patients struggle with the flexion phase of postural transition compared to healthy older adults. Buckley et. al documented that healthy older adults (1.55-2.09 s) take a greater amount of time to complete the STW task compared to healthy young adults (1.36-1.56 s). ${ }^{10}$ Furthermore, Kerr et al. ${ }^{29}$ observed that older adults with postural instability, completed the STW task slower than their age matched controls. Accordingly, our results found significant differences in STW completion times of post-HD and pre-HD, following dialysis therapy, patients took approximately $15.2 \%$ longer than they did before the dialysis therapy. Thus, it appears that the patients with ESRD on HD struggle to complete even inherent everyday functional tasks after the HD session.

\section{Event detection and phase description of STW movement}

An important part of this study was the identification of distinct events during STW movements. The identified events were consistent for all subjects and were ordered in following sequences: (1) Initiation (2) Peak Flexion Velocity (3) Seat Off Event (4) Peak Extension Velocity (5) GI (6) Swing Foot Toe-Off (7) Swing Foot Heel-Strike (8) Stance Foot Toe-Off (9) Stance Foot Heel-Strike (Fig. 2).

A similar work by Kerr et al., ${ }^{29,30}$ defined four phases in STW movements: Phase 1 of flexion momentum ends at the time of "seat-off"; Phase 2 of extension momentum begins at the time of seat-off and ends when the peak vertical velocity is reached; Phase 3 of unloading begins with GI and is characterized by lateral weight transfer using kinetic information; phase 4 corresponds to the first step starting with swing toe-off and ending with stance toe-off. Buckley et al. ${ }^{10,11}$ was not able to duplicate the GI event defined by Kerr et al., ${ }^{29,30}$ hence, redefined initiation of gait in STW as the instant of swing leg heel-off. In particular, computation of peak vertical COM velocity limits the study to camera based laboratories. GI event description with force plates allows parsing of the antero-posterior and medio-lateral events. However, as a result of trunk extension, the GI preparatory phase (swing foot unloading), and the biomechanical events that characterize it, phase 2 is merged with phase 3, and subsequently decreases the complexity of interpretation of the temporal sequence of phases separately. Therefore, by introducing a combined extension and unloading phase as phase 2 in our study, we have removed complexity involved in peak COM vertical velocity event detection and the GI event; hence, we effectively simplified the STW phase classification. This simplified approach involving combined extension and unloading phase has although reduced resolution of STW measurement prior to initiation of gait in the STW sequence. Moreover, the benefits of combining the STW movement phases is that an on-body sensor can easily capture this information, making STW measurement 
procedure potentially accessible to clinicians in identifying fall risk. ${ }^{30}$ Accordingly, while the limited literature suggests falls are particularly problematic for elderly HD patients, ${ }^{13,15,27}$ a better understanding of functional activities such as STW could provide useful information to improve interventions for falls in HD treated population.

\section{Limitations}

Conclusions based on this study should be considered in the context of its limitations. Although every attempt was made to standardize the patient's movement by using a standard arm-chair of fixed height, patients used backrest to sit with their thighs and foot parallel to each other. We did not record the strategy of rising from chair but was determined by postprocessing IMU data placed on wrists. Although this does not alter the natural pattern of movement, the magnitude of trunk angular velocity and initial flexion acceleration are different in each strategy, thus patients had been instructed to raise themselves using armrest always. Previous studies on STW have defined GI as the peak in medio-lateral shear forces, when patient's feet are placed on force plates; and were not sensitive enough to get detected in most of the trials. However, in our simplified approach, combining both extension and unloading phases, we lose the information applicable to gait commencement in the STW sequence. In addition, our study lacks control data; thus, the experimental design is limited to whether impairment of STW is a result of sitting in a chair for $4 \mathrm{~h}$. However, our data did not show systematic improvements in post-HD trials, permitting us to rule out the idea that impairment in the STW movement was simply due to sitting in a chair for $4 \mathrm{~h}$. Similarly, the results in our preliminary study cannot be generalized for the elderly ESRD population, because we only tested six patients and their average age was less than 60 years. Lastly, to reduce intra-subject variability, we did not conduct warm-up trials of the task in both pre- and post-HD data collection.

\section{CONCLUSION}

The present study demonstrates the potential of an IMU to measure STW performance to detect and quantify subtle abnormalities in mobility, balance, coordination and functional abilities. In clinical settings, the mobility parameters and characteristics examined in this study could provide further insights into motor performance of patients after dialysis treatment and may provide an evaluation standard for fall risk in these patients. The clinical utility and relevance for using IMU's is in its continuous (approximately $22 \mathrm{~h}$ ) data acquisition and analysis after dialysis treatment, because HD is a cyclic therapy (considering thrice weekly HD regimen) and time for occurrence of abnormal postural transitions resulting in falls is uncertain. Our results indicate that ESRD patients rise slower after dialysis; this may be due to combined deterioration effects of dialysis on neuromuscular function and reduced strength. STW analysis could improve our ability to identify HD patients at risk of falls, to document effects of dialysis therapy in this perspective, and to assess the response and benefits with other therapeutic interventions. As fall interventions are effective, screening HD patients for falls may become important. On identification of specific component of STW movement which is problematic, physicians may be able to refine the rehabilitation programme. Currently, subjective ratings of functional movements are standard feature of clinical mobility tests. This study has potential to shift current clinical mobility assessment paradigms with an immediate impact because this type of objective assessments (afforded by the novel mobile sensors) can be easily implemented inmost clinical settings. The results of this preliminary study have little implications for targeted rehabilitation programs in patients after HD therapy; however, future research with more participants may help in designing specialized rehabilitation programs. Furthermore, this study enhances understanding of daily activities such as STW movement and provides a model for screening patients at risk of fall. 


\section{Acknowledgments}

This research was supported by the NSF (Grant \#CBET-0756058) and NIOSH (grant \#CDC/NIOSHR01OH009222). Additionally, supported by the NIH (L30-AG022963-04/NIH HHS/United States). Authors are also thankful to Sharon (Lisa) Johnson, Adam Barth and Xiaoyue Zhang for data collection.

\section{REFERENCES}

1. Abdel-Rahman EM, Turgut F, Turkmen K, Balogun RA. Falls in elderly hemodialysis patients. QJM. 2011; 104:829-838. [PubMed: 21750022]

2. Abdel-Rahman EM, Yan G, Turgut F, Balogun RA. Long-term morbidity and mortality related to falls in hemodialysis patients: role of age and gender-a pilot study. Nephron Clin. Pract. 2011; 118:c278-c284. [PubMed: 21212691]

3. Aberg AC, Frykberg GE, Halvorsen K. Mediolateral stability of sit-to-walk performance in older individuals with and without fear of falling. Gait Posture. 2010; 31:438-443. [PubMed: 20189390]

4. Barth, AT.; Hanson, MA.; Powell, HC., Jr; Lach, J. International Workshop on Wearable and Implantable Body Sensor Networks. Cambridge: 2009. TEMPO 3.1: a body area sensor network platform for continuous movement assessment; p. 71-76.

5. Berchtold MW, Brinkmeier H, Muntener M. Calcium ion in skeletal muscle: its crucial role for muscle function, plasticity, and disease. Physiol. Rev. 2000; 80:1215-1265. [PubMed: 10893434]

6. Bohannon RW, Hull D, Palmeri D. Muscle strength impairments and gait performance deficits in kidney transplant candidates. Am. J. Kidney Dis. 1994; 24:480-485. [PubMed: 8079973]

7. Bouten CV, Koekkoek KT, Verduin M, Kodde R, Janssen JD. A triaxial accelerometer and portable data processing unit for the assessment of daily physical activity. IEEE Trans. Biomed. Eng. 1997; 44:136-147. [PubMed: 9216127]

8. Brouns R, De Deyn PP. Neurological complications in renal failure: a review. Clin. Neurol. Neurosurg. 2004; 107:1-16. [PubMed: 15567546]

9. Brunt D, Lafferty MJ, McKeon A, Goode B, Mulhausen C, Polk P. Invariant characteristics of gait initiation. Am. J. Phys. Med. Rehabil. 1991; 70:206-212. [PubMed: 1878180]

10. Buckley T, Pitsikoulis C, Barthelemy E, Hass CJ. Age impairs sit-to-walk motor performance. J. Biomech. 2009; 42:2318-2322. [PubMed: 19656512]

11. Buckley TA, Pitsikoulis C, Hass CJ. Dynamic postural stability during sit-to-walk transitions in Parkinson disease patients. Mov. Disord. 2008; 23:1274-1280. [PubMed: 18464285]

12. CDC (Centers for Disease Control and Prevention). Center for Injury Prevention and Control. Atlanta: CDC; 2010.

13. Cook WL, Tomlinson G, Donaldson M, Markowitz SN, Naglie G, Sobolev B, Jassal SV. Falls and fall-related injuries in older dialysis patients. Clin. J. Am. Soc. Nephrol. 2006; 1:1197-1204. [PubMed: 17699348]

14. Debold EP. Recent insights into muscle fatigue at the cross-bridge level. Front Physiol. 2012; 3:151. [PubMed: 22675303]

15. Desmet C, Beguin C, Swine C, Jadoul M. Université Catholique de Louvain Collaborative Group. Falls in hemodialysis patients: prospective study of incidence, risk factors, and complications. Am. J. Kidney Dis. 2005; 45:148-153. [PubMed: 15696454]

16. Diesel W, Noakes TD, Swanepoel C, Lambert M. Isokinetic muscle strength predicts maximum exercise tolerance in renal patients on chronic hemodialysis. Am. J. Kidney Dis. 1990; 16:109114. [PubMed: 2382645]

17. Dion L, Malouin F, McFadyen B, Richards CL. Assessing mobility and locomotor coordination after stroke with the rise-to-walk task. Neurorehabil. Neural Repair. 2003; 17:83-92. [PubMed: 12814053]

18. Fahal IH, Bell GM, Bone JM, Edwards RHT. Physiological abnormalities of skeletal muscle in dialysis patients. Nephrol. Dial. Transplant. 1997; 12:119-127. [PubMed: 9027785]

19. Flandrin P, Rilling G, Goncalves P. Empirical mode decomposition as a filter bank. IEEE Signal Process. Lett. 2004; 11:112-114. 
20. Fordyce AM, Lalani Z, Songra AK, Hildreth AJ, Carton AT, Hawkesford JE. Intermaxillary fixation is not usually necessary to reduce mandibular fractures. Br. J. Oral Maxillofac. Surg. 1999; 37:52-57. [PubMed: 10203223]

21. Greene BR, O’Donovan A, Romero-Ortuno R, Cogan L, Scanaill CN, Kenny RA. Quantitative falls risk assessment using the timed up and go test. IEEE Trans. Biomed. Eng. 2010; 57:29182926. [PubMed: 20923729]

22. Halliday SE, Winter DA, Frank JS, Patla AE, Prince F. The initiation of gait in young, elderly, and Parkinson's disease subjects. Gait Posture. 1998; 8:8-14. [PubMed: 10200394]

23. Hass CJ, Waddell DE, Wolf SL, Juncos JL, Gregor RJ. Gait initiation in older adults with postural instability. Clin. Biomech. (Bristol, Avon.). 2008; 23:743-753.

24. Headley S, Germain M, Mailloux P, Mulhern J, Ashworth B, Burris J, Brewer B, Nindl B, Coughlin M, Welles R, Jones M. Resistance training improves strength and functional measures in patients with End-Stage Renal Disease. Am. J. Kidney Dis. 2002; 40:355-364. [PubMed: 12148109]

25. Huang NE, Shen Z, Long SR. A new view of nonlinear water waves: the Hilbert spectrum. Annu. Rev. Fluid Mech. 1999; 31:417-457.

26. Huang NE, Shen Z, Long SR, Wu MC, Shih HH, Zheng Q, Yen NC, Tung CC, Liu HH. The empirical mode decomposition and the Hilbert spectrum for nonlinear and non-stationary time series analysis. Proc. R. Soc. A Math. Phys. Eng. Sci. 1998; 454:903-995.

27. Jassal SV, Cook WL. Prevalence of falls among seniors maintained on hemodialysis. Int. Urol. Nephrol. 2005; 37:649-652. [PubMed: 16307356]

28. Johansen KL, Shubert T, Doyle J, Soher B, Sakkas GK, Kent-Braun JA. Muscle atrophy in patients receiving hemodialysis: effects on muscle strength, muscle quality, and physical function. Kidney Int. 2003; 63:291-297. [PubMed: 12472795]

29. Kerr A, Durward B, Kerr KM. Defining phases for the sit-to-walk movement. Clin. Biomech. 2004; 19:385-390.

30. Kerr A, Rafferty D, Kerr KM, Durward B. Timing phases of the sit-to-walk movement: validity of a clinical test. Gait Posture. 2007; 26:11-16. [PubMed: 16908153]

31. Large J, Gan N, Basic D, Jennings N. Using the timed up and go test to stratify elderly inpatients at risk of falls. Clin. Rehabil. 2006; 20:421-428. [PubMed: 16774093]

32. Li M, Tomlinson G, Naglie G, Cook WL, Jaassal SV. Geriatric comorbidities, such as falls, confer an independent mortality risk to elderly dialysis patient. Nephrol. Dial. Transplant. 2007; 23(4):15.

33. Li M, Tomlinson G, Naglie G, Cook WL, Jassal SV. Geriatric comorbidities, such as falls, confer an independent mortality risk to elderly dialysis patients. Nephrol. Dial. Transplant. 2008; 23:1396-1400. [PubMed: 18057068]

34. Lockhart TE, Barth AT, Zhang X, Songra R, Abdel-Rahman E, Lach J. Portable, non-invasive fall risk assessment in end stage renal disease patients on hemodialysis. ACM Trans. Comput. Hum. Interact. 2010:84-93. [PubMed: 22124286]

35. Lockhart TE, Shi W. Effects of age on dynamic accommodation. Ergonomics. 2010; 53:892-903. [PubMed: 20582770]

36. Lord SR, Murray SM, Chapman K, Munro B, Tiedemann A. Sit-to-stand performance depends on sensation, speed, balance, and psychological status in addition to strength in older people. J. Gerontol. A Biol. Sci. Med. Sci. 2002; 57:M539-M543. [PubMed: 12145369]

37. Magnan A. Modification of the sit-to-stand task with the addition of gait initiation. Gait Posture. 1996; 4:232-241.

38. Mathias S, Nayak US, Isaacs B. Balance in elderly patients: the "get-up and go" test. Arch. Phys. Med. Rehabil. 1986; 67:387-389. [PubMed: 3487300]

39. Oliver D. Risk factors and risk assessment tools for falls in hospital in-patients: a systematic review. Age Ageing. 2004; 33:122-130. [PubMed: 14960426]

40. Painter P, Carlson L, Carey S, Paul S, Myll J. Physical functioning and health-related quality-oflife changes with exercise training in hemodialysis patients. Am. J. Kidney Dis. 2000; 35:482-492. [PubMed: 10692275] 
41. Pate E, Bhimani M, Franks-Skiba K, Cooke R. Reduced effect of $\mathrm{pH}$ on skinned rabbit psoas muscle mechanics at high temperatures: implications for fatigue. J. Physiol. 1995; 486(Pt 3):689694. [PubMed: 7473229]

42. Podsiadlo D, Richardson S. The timed "Up \& Go": a test of basic functional mobility for frail elderly persons. J. Am. Geriatr. Soc. 1991; 39:142-148. [PubMed: 1991946]

43. Saiki JK, Vaziri ND, Naeim F, Meshkinpour H. Dialysis-induced changes in muscle strength. J. Dialysis. 1980; 4:191-201.

44. Sharma PK, Songra AK, Ng SY. Intraoperative ultrasound-guided retrieval of an airgun pellet from the tongue: a case report. Br. J. Oral Maxillofac. Surg. 2002; 40:153-155. [PubMed: 12180211]

45. Stehman-Breen C, Alem AM, Sherrard DJ, Gillen DL, Weiss NS, Beresford SA, Heckbert SR, Wong C. Increased risk of hip fracture among patients with end-stage renal disease. Kidney Int. 2000; 58:396-399. [PubMed: 10886587]

46. Stevens JA, Olson S. Reducing falls and resulting hip fractures among older women. MMWR Recomm. Rep. 2000; 49:3-12. [PubMed: 15580729]

47. Weiss A, Herman T, Plotnik M, Brozgol M, Maidan I, Giladi N, Gurevich T, Hausdorff JM. Can an accelerometer enhance the utility of the Timed Up \& Go Test when evaluating patients with Parkinson's disease? Med. Eng. Phys. 2010; 32:119-125. [PubMed: 19942472]

48. Whitney SL, Marchetti GF, Schade A, Wrisley DM. The sensitivity and specificity of the timed "Up \& Go" and the dynamic gait index for self-reported falls in persons with vestibular disorders. J. Vestib. Res. 2004; 14:397-409. [PubMed: 15598995]

49. Wu Z, Huang NE. Ensemble empirical mode decomposition: a noise-assisted data analysis method. Adv. Adapt. Data Anal. 2009; 01:1. 
(a)

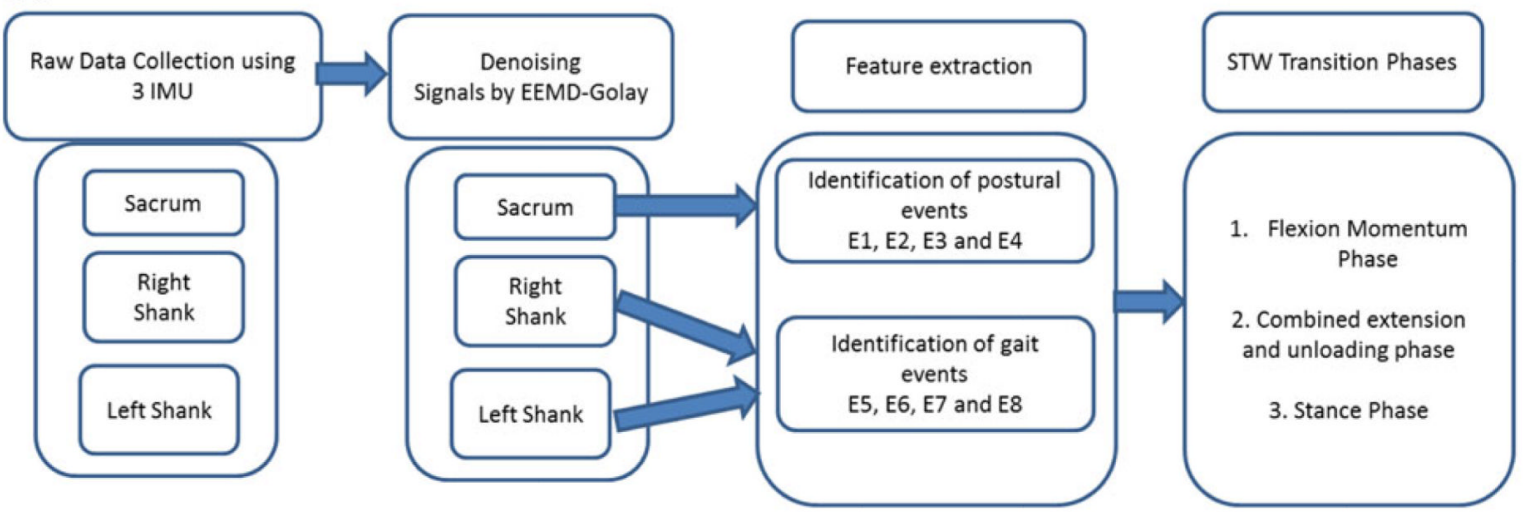

(b)

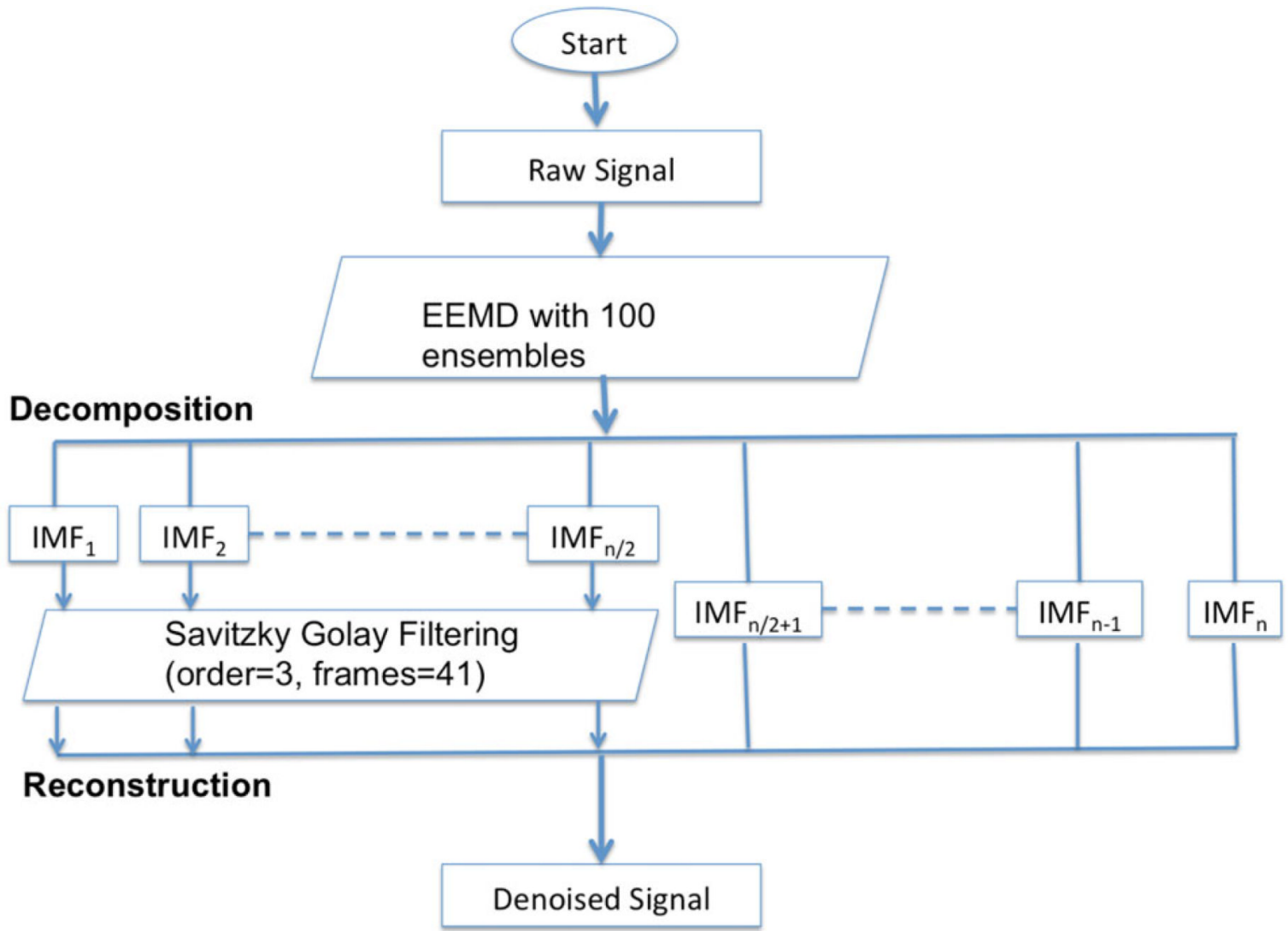

FIGURE 1.

(a) Schematic diagram of procedure for signal conditioning and STW phase classification.

(b) Schematic diagram of denoising signals by EEMD-Golay method. 


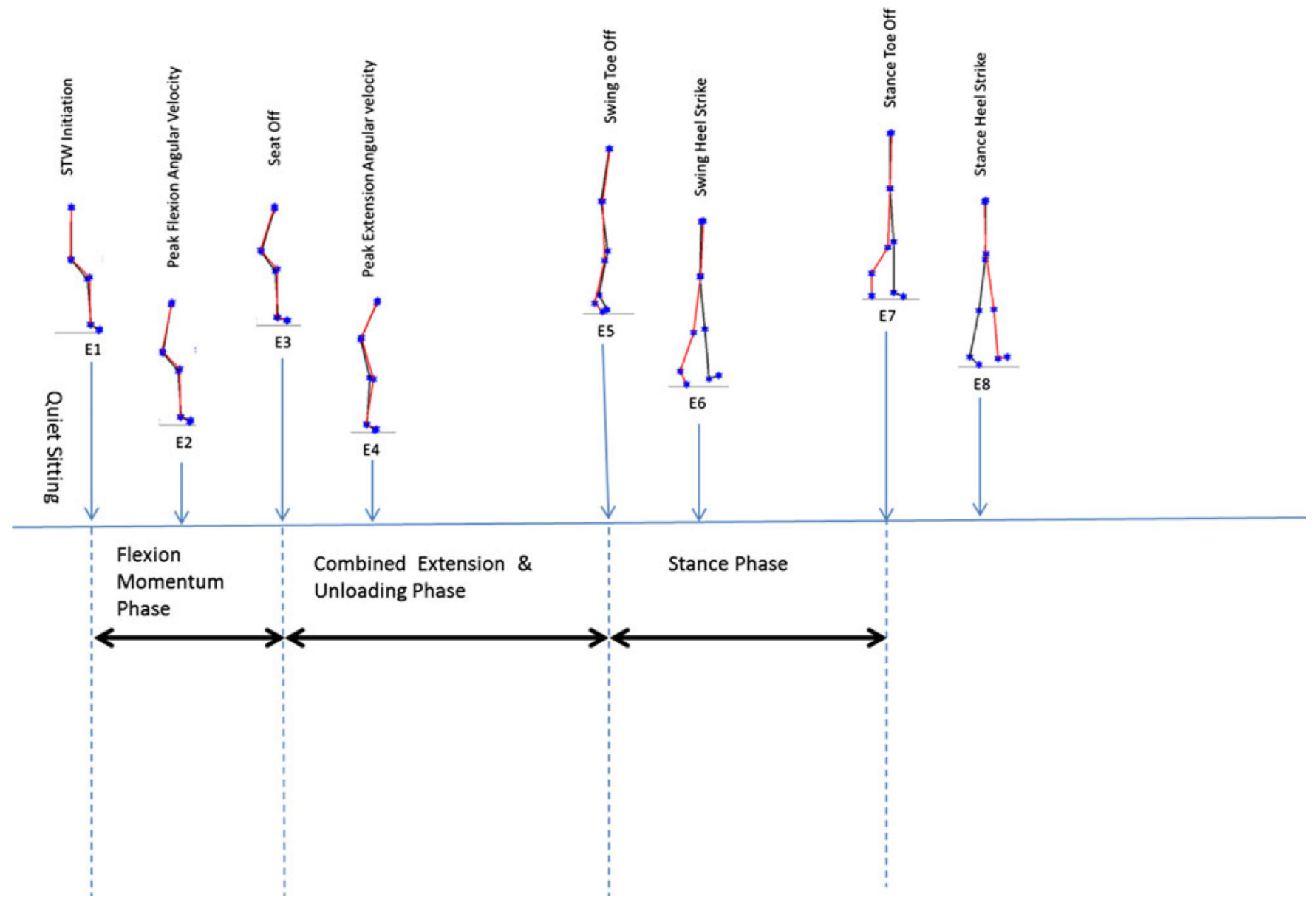

FIGURE 2.

Three phases of STW (1) Flexion momentum phase, (2) Combined extension and unloading phase, and (3) Stance phase and eight postural transition and gait events detected using denoised IMU signals (E1-E8). 

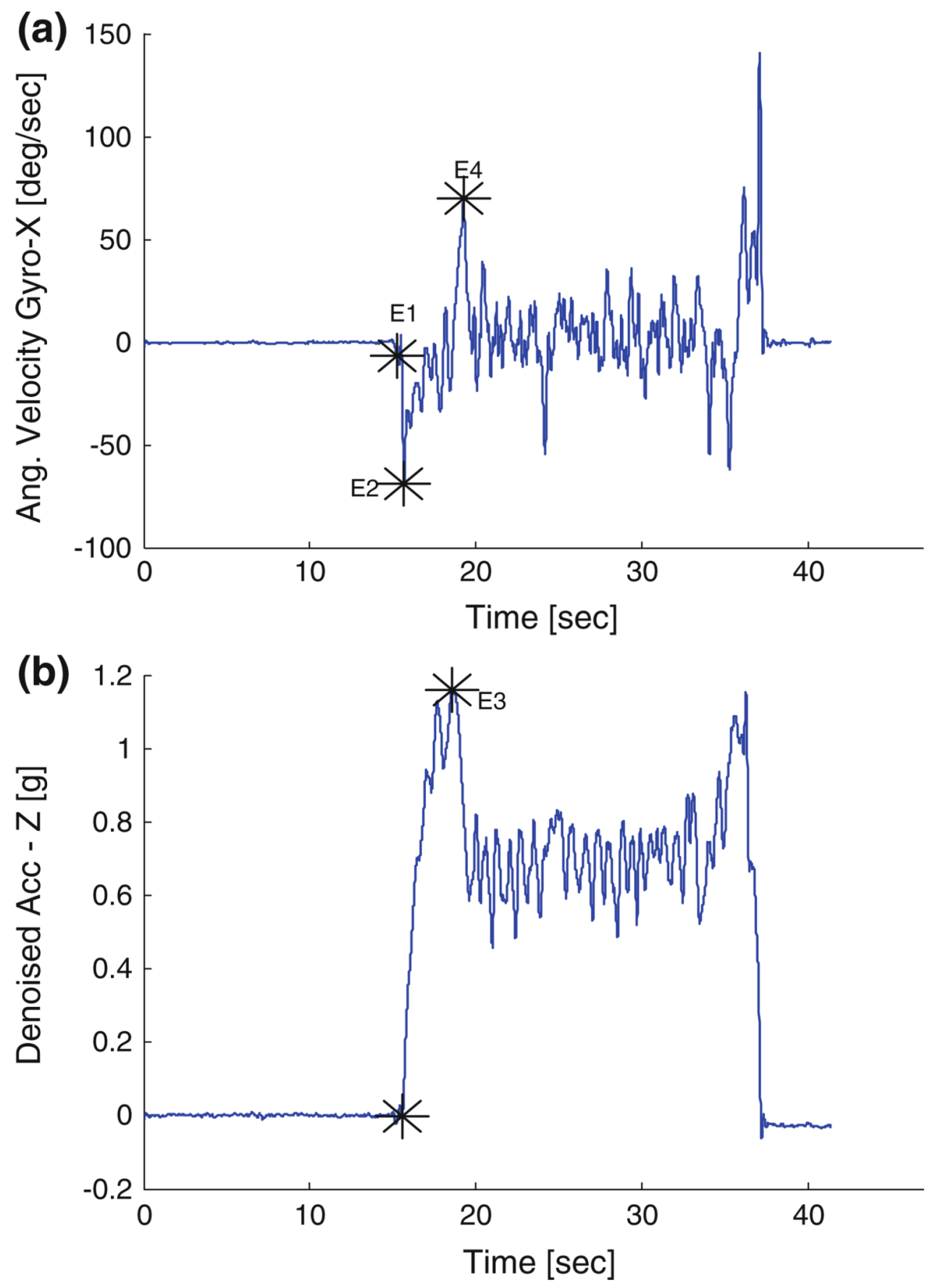

FIGURE 3.

Typical example of gyroscope (Gyro X) and accelerometer (Acc Z) signals of a sample subject (IMU located at sacrum). Event detection: (a) STW Initiation (E1), Peak flexion angular velocity (E2), Peak extension angular velocity (E4) and (b) seat off event (E3). 

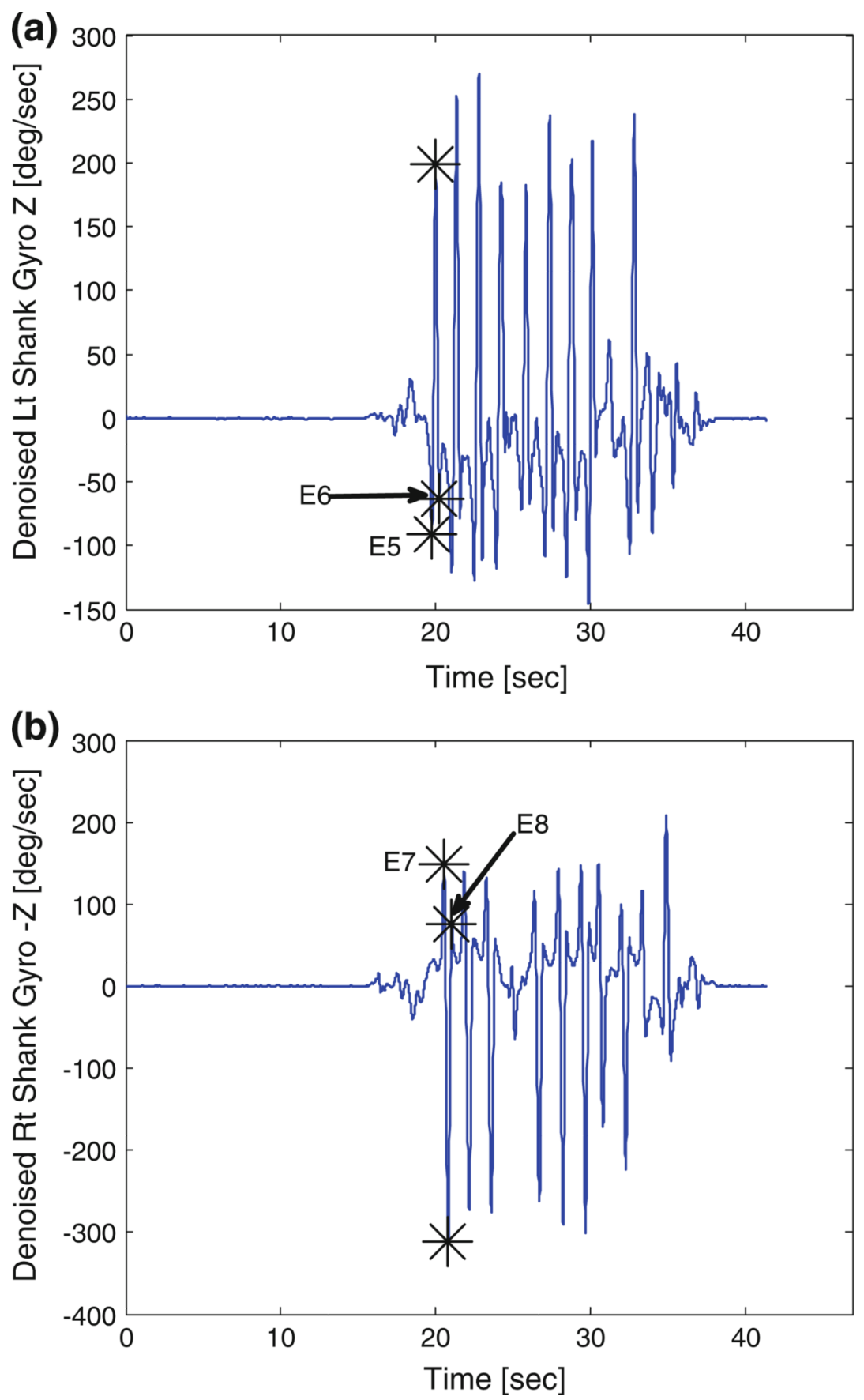

FIGURE 4.

Typical examples of gyroscope (Gyro Z) signals of a sample subject (IMU located at lateral side of both shanks). Event detection from swing (left) and stance (left) foot sensors. (a) swing foot and corresponding toe off event (E5) and heel strike event (E6). (b) stance foot and corresponding toe off event (E7) and heel strike event (E8). 


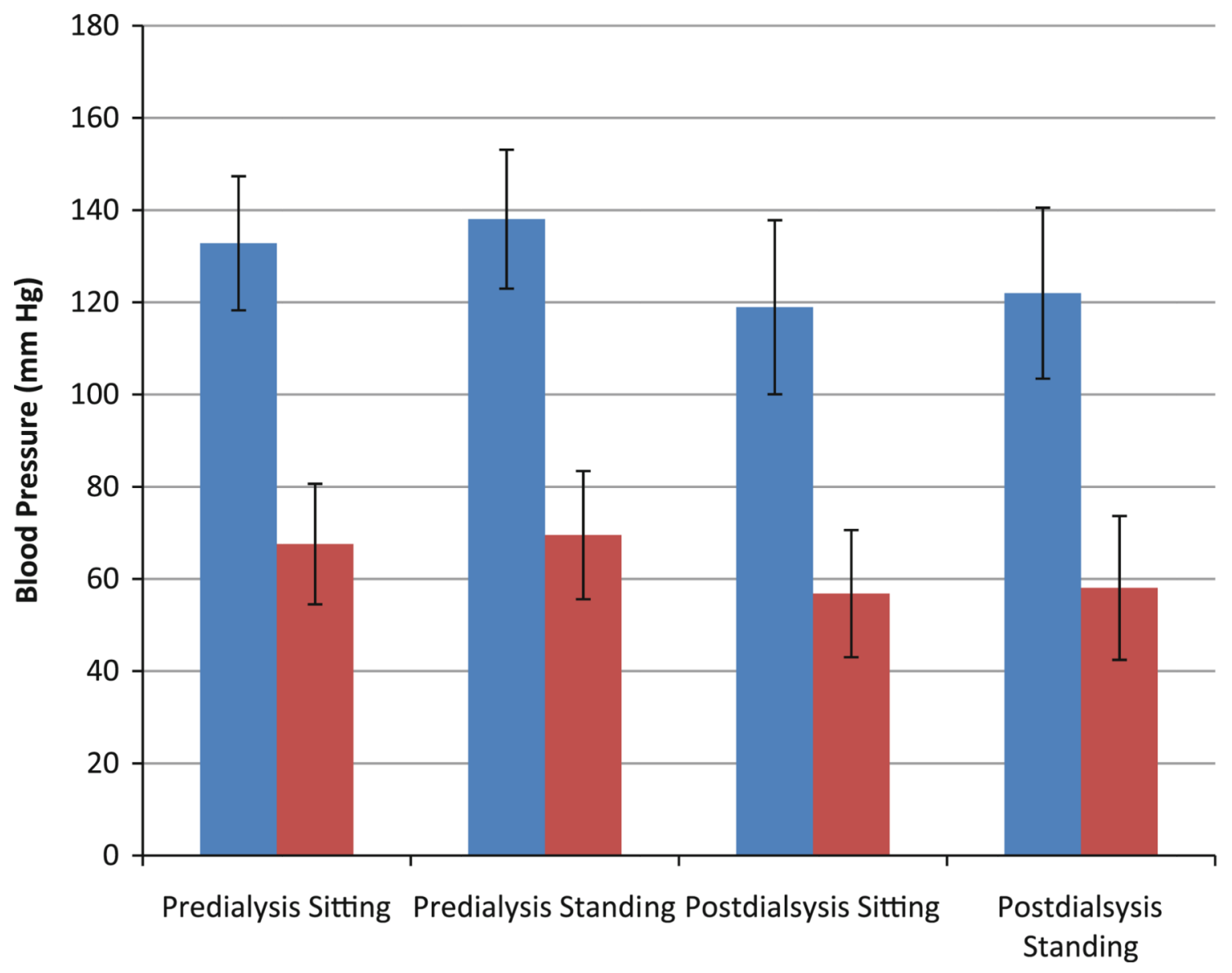

FIGURE 5.

Mean $( \pm \mathrm{SD})$ changes of blood pressure during sitting and standing for pre- and post-dialysis. 


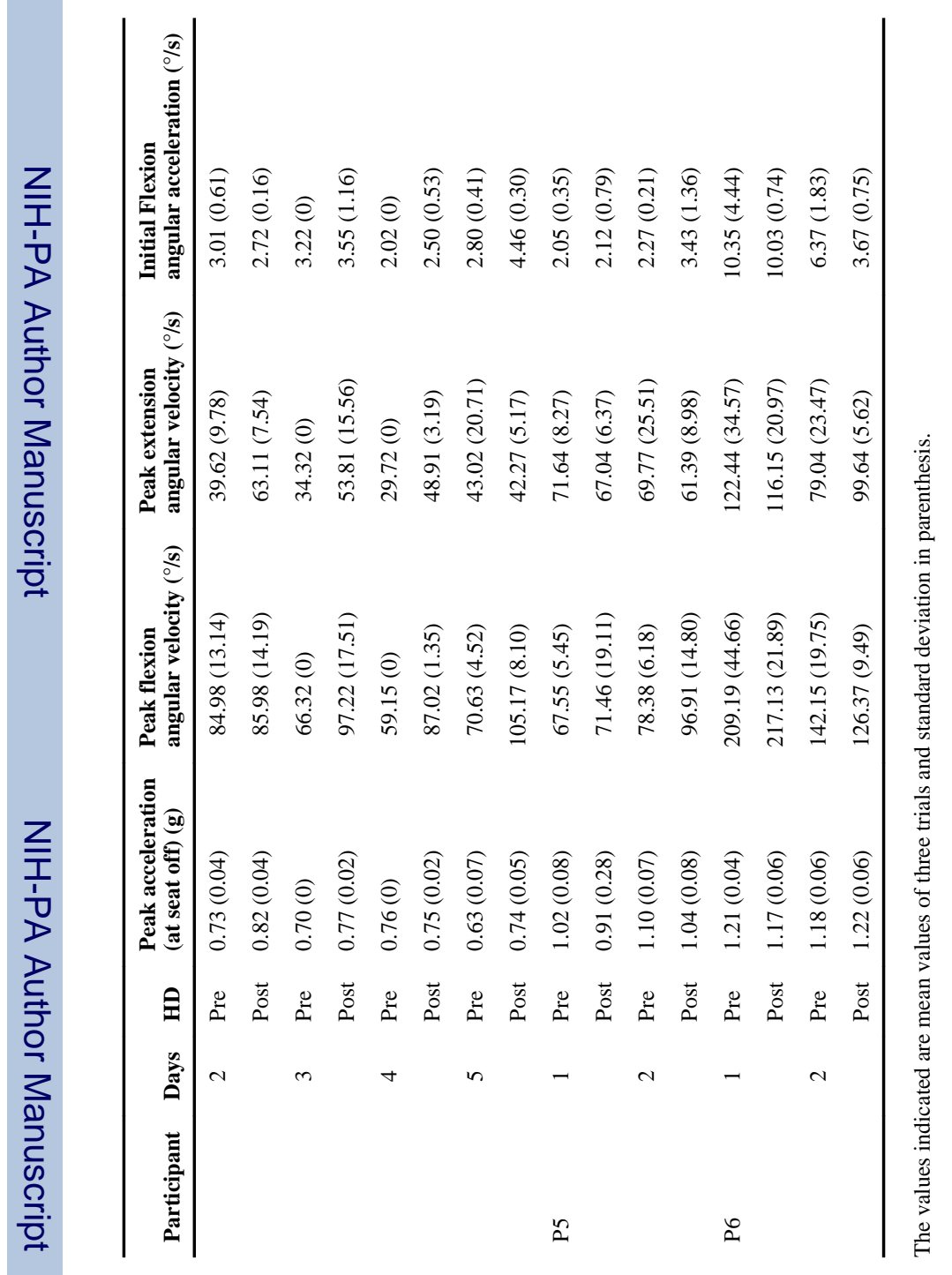


TABLE 2

Time interval to reach peak flexion and extension events and the time taken to complete three phases and complete STW task.

\begin{tabular}{lccl}
\hline Time between events & Pre HD $(\mathbf{s})$ & Post HD $(\mathbf{s})$ & $\boldsymbol{p}$ value \\
\hline STW initiation to peak flexion(E1-E2) & $0.47(0.04)$ & $0.80(0.07)$ & $0.03^{*}$ \\
STW initiation to peak extension (E1-E4) & $1.53(0.19)$ & $1.91(0.21)$ & $0.02^{*}$ \\
Phase 1 (E1-E3) & $1.04(0.14)$ & $1.36(0.17)$ & $0.04^{*}$ \\
Phase 2 (E5-E3) & $0.84(0.14)$ & $0.79(0.00)$ & 0.72 \\
Phase 3 (E7-E5) & $0.57(0.07)$ & $0.73(0.04)$ & 0.14 \\
STW completion (E7-E1) & $2.46(0.57)$ & $2.90(0.89)$ & $0.04^{*}$ \\
First double support (E7-E6) & $0.14(0.10)$ & $0.29(0.32)$ & 0.24 \\
\hline
\end{tabular}

The values indicated are mean values of three trials and standard deviation in parenthesis. 